\title{
Robot Topological Map Generation from Formal Route Instructions
}

\author{
Mohammed Elmogy ${ }^{1}$ and Christopher Habel ${ }^{1}$ and Jianwei Zhang ${ }^{1}$
}

\begin{abstract}
Mobile robots and Humanoids need to use spatial information about their surrounding environment in order to effectively plan and execute navigation tasks. This spatial information can be presented in various ways to increase the interaction between the human and the robot. One of the more effective ways is by describing the route verbally which bridges the gap between the forms of spatial knowledge of such robots and the forms of language used by humans.

In this paper, we build a topological map for robot route description. This map represents the route's motion actions and spatial relationships graphically to plan robot's navigation task. The map is generated by using Formal Route Instructions (FRIs) to simplify the route description process and also to avoid ambiguity. FRIs are designed to be simple, easy to use, and suitable for naïve users.
\end{abstract}

\section{INTRODUCTION}

A robot is an intelligent, multi-purpose machine, which can carry out a variety of online tasks. An essential aspect, which distinguishes robotics from other areas of AI, is the interaction of robots with humans and with their surrounding environment. In a robot system, various autonomous components such as sensing, recognition, planning, control, and their coordination must cooperate in recognizing the environment, solving problems, planning a behavior, and executing it. In order to make this interaction more intelligent, a robot needs functions such as: the ability to understand the environment by visual recognition, the ability to perform dexterous manipulation using force, tactile, and visual feedback, the ability to plan task procedures, the ability to communicate with humans, the ability to learn how to perform tasks, the ability to recover from errors, and so on [1]. All of these functions are required for robot intelligence to be realized adequately.

Due of the potential for interaction with humans, research in humanoid robotics has made significant progress in recent years. The key reason for preferring humanoids is their shape, which seems to be optimal for being taught by humans and learning from humans. Humanoid robotics labs worldwide are working on creating robots that are similar to humans in shape and behavior. These similarities have been proven to facilitate the

\footnotetext{
1 Department of Informatics, University of Hamburg, \{elmogy, habel, zhang\}@informatik.uni-hamburg.de
}

communication task between the human and the robot. Recent studies also show additional advantages of humanoids which can be summarized into three points. The first advantage is that human interaction with robots is easier if robots are humanoid because of its shape. The second is that acceptance of robots by humans is easier for those with humanoid shape. The last advantage is that the efficiency of teaching and programming a robot is highest with humanoids [2-4].

A more natural interaction between the humans and the humanoid can be achieved if there are ways to bridge the gap between the forms of spatial knowledge of such robots and the forms of language used by humans, enabling them to communicate by using this shared knowledge. A natural language interface supports more 'natural' styles of interaction between robots and users. Most typical scenarios include a human user instructing a robot to perform some actions in these scenarios, such as moving to a location or manipulating an object. Route descriptions, which are used to guide the robot in executing navigation tasks in the surrounding environment, are considered as one of the most important natural language interfaces between human and robots in realizing effective human-robot interaction.

The remaining part of this paper is structured as follows. In the next section, robot navigation is elucidated. How to generate good route instructions and what are the different broad categories of route-based navigation in robotics are presented in details. In section 3, the main building blocks of our system architecture is explained. The route processing module is discussed in details with concentrating on how the Formal Route Instructions are used to describe the user's route to the robot. Also, the topological map representation of the route description is introduced.

\section{ROBOT NAVIGATION}

Navigation has always been an interdisciplinary topic of research, because mobile agents of different types are inevitably faced with similar navigational problems. Therefore, human navigation can readily be compared to the navigation of other biological organisms or of artificial mobile agents like mobile robots. Thus navigation can be described as a coordinated and goal-directed movement through the environment by biological organisms or intelligent machines. It involves both the planning and execution of movements.

Following Montello [5] we consider navigation to consist of two components: locomotion and wayfinding. Locomotion is the movement of the agents's body around an environment, 
coordinated specifically to the local or close surroundings. There are various modes of locomotion which are important because they determine much about the way we acquire and process information as we move. Thus, with respect to humans 'locomotion' concerns the level of motor processes and automatic (or unconscious) cognitive and sensor processes. On the other hand, wayfinding refers to the goal-directed and planned movement of a body around an environment in an efficient way. It requires a goal locality, a destination the agent wants to reach. A wayfinding action such as following verbal directions clearly requires the activation of longterm knowledge representations (the cognitive map) into working memory in order to access one's knowledge of place layouts $[5,6]$. Thus, 'wayfinding' concerns higher cognitive and communicative processes. The great majority of acts of navigation involve both locomotion, and wayfinding components. Evidence for the distinction's validity is provided by the simple fact that you can have one without the other. They are generally components of an integrated system of navigation that can be separated only conceptually, but sometimes they can be separated literally.

With respect to robots, their navigation system is based on three basic components. The first is planning, which computes a trajectory or path between two points (starting and end points). Note that path planning for robots has to include both levels, wayfinding and locomotion. The second component-often also called navigation-provides the robot with those pieces of information needed to move and to follow the computed path/trajectory. The last component is environment representation, which enables the robot to know its location and its heading. For indoor robot navigation, systems are classified into three groups: map-based navigation using predefined geometric and/or topological models, map-building-based navigation constructing geometric and/or topological models on its own, and mapless navigation using only object recognition and actions associated to these objects [7].

In vision based robot navigation systems, vision sensors (cameras) are used to provide a huge amount of data that should be processed in real time. The elements extracted from the data are compared to reference models which are stored previously as knowledge database. In these systems, they concentrate on the field of shape understanding using the data captured from the vision sensors. Environment interpretation stresses the use of natural landmarks to ease the navigation and the pose estimation of a mobile robot.

\subsection{Robot Route Instructions}

Route instructions specify spatial information about the environment of the route and temporal information about the actions (movements, turns) to be performed. Human route instructions are usually conveyed either verbally in spoken discourse or written texts, or by graphical means, i.e. by illustrating the route on a map, or drawing sketch-maps. A third possibility is to combine these two kinds of external representations leading to multimodal route instructions. Whereas verbal route instructions focus on the actions to be performed and take the spatial environment as the frame for these actions, maps and other pictorial representations foreground the spatial environment without possessing adequate means for representing the actions [8]. Nevertheless, all type of route instructions have to provide correlated actions, paths, tracks, positions and landmarks to describe the navigation path to the navigating agent. All of these route instruction components can be classified and categorized into main groups to facilitate the analysis of the navigation task [9].
Good route instructions should contain adequate information about the following two aspects. The first aspect concerns navigation actions, in particular locomotion actions and perception actions, which are performed by the robot to reach its destination. The second is the spatial environment, in which the intended locomotion of the robot will take place. The instructor's primary task is to choose a good combination of communicational means to transfer the relevant information concerning both aspects to the robot [8].

MacMahon [10] proposes four basic actions to be used in following route instructions: turning in place, moving from one place to another, verifying a view description against an observation and terminating a current action. The primary characteristic of a path is the change of location. Turns can be viewed as changes in orientation. These considerations led to four basic types of Navigational Information: moves, turns, positions and orientations. Altogether, moves and turns can be subsumed under the general notion of actions, and positions and orientations can be viewed as verifications.

The field of route-based robot navigation is regularly be classified into four categories. The first category is Guidance, which is mainly concerned with directly leading an agent by external cues - either by following a particular gradient or moving to match the current sensory image with a stored image of the target or of the surroundings. In all of these cases, the robot tries to locally maximize a predefined criterion without knowledge of spatial relations in the environment or about its own position. The second is the Guidance Place Recognition - Triggered Response. For place recognition based strategies, complex spatial behaviors are triggered at distinct points in space. Once the correct place is recognized, the associated action (e.g., movement in a particular direction or guided behavior) will lead to complex trajectories. The main problem of this strategy obviously consists in the correct identification of a place. The third category is Topological Navigation, which describes navigation based on topological networks, is thus a more flexible extension of place-triggered navigation. The basic elements of this type of networks are places and some connections between these places. Finally, the last category is the Metrical Navigation. Unlike the last two approaches, which divide space into a small number of distinct places and the space in-between, metrical navigation does not require such a distinction in principle. The metric most frequently used is Euclidean, thus distances and angles are well defined and can be used to drive spatial navigation. Pre-existing maps, which specify the metrical relations between objects in the environment of the agent, are often supplied directly or are autonomously constructed by triangulation and integration of sensory information $[11,12]$.

\subsection{Topological and Metric Maps for Robots}

Building a representation of the environment is an essential task for a mobile robot that aims at moving autonomously in the surrounding space. The representation of spatial knowledge can be considered at two different levels of abstraction. On the one hand, metric (geometric) maps represent the environment according to the absolute geometric position of landmarks. On the other hand, a topological map is a more abstract representation that describes relationships among features of the environment, without any absolute reference system [13].

Approaches in the metric paradigm generate fine-grained, metric descriptions of a robot's environment. In these representations, the robot's environment is defined by a single global coordinate system, in which all mapping and navigation 
takes place. Typically, the metric map is structured by a grid with each cell of the grid representing some amount of space in the real world. These grids become quite sophisticated at representing the spatial structure of the world.

On the other hand, approaches in the topological paradigm generate coarse, graph-like descriptions of environments, where nodes correspond to significant, easy-to-distinguish places or landmarks, and arcs correspond to actions or action sequences that connect neighboring places. Topological maps are qualitative descriptions of the robot's workspace, in which the environment is represented as places and connections between places. Topological maps can also be more compact in their representation of space, in that they represent only interesting places and not the entire environment. Topological maps have been proved as very successful for mobile robots [10].

In principle, topological maps could be scale to the size of large-scale indoor environments better than metric maps could, because a coarse-grained, graph-structured representation is much more compact than a dense array, and more directly suited to problem solving algorithms. However, purely topological maps have difficulty in distinguishing adequately between different places, and have not been applied to large environments in practice. Recent progress in metric mapping has made it possible to build useful and accurate metric maps of reasonable large scale environments, but memory and time complexity pose serious problems [14].
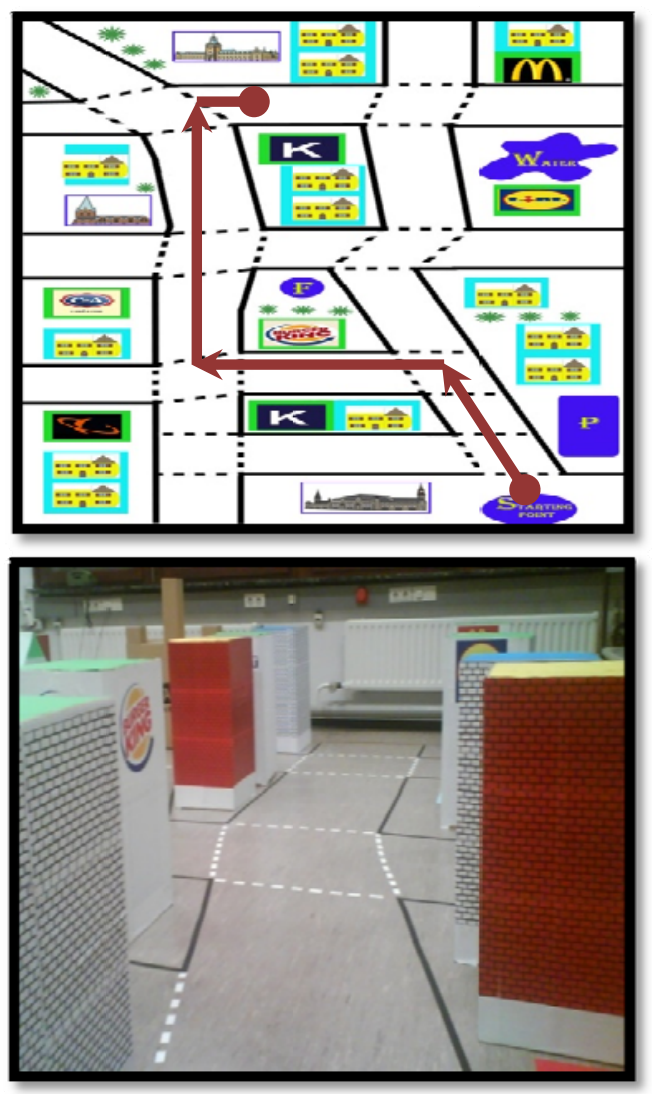

Figure 1. Humanoid miniature city. Top: Layout of the miniature city with an exemplary route (fat line), whose verbal description is discussed below. Bottom: Physical realization of the miniature city.

When robots navigate in indoor environments, it requires an adequate representation of the working space. This representation should be abstract enough to facilitate higher-level reasoning tasks like strategic planning or situation assessment, and still be detailed enough to allow the robot to perform lower-level tasks like path planning/navigation or self-localization. A common belief in the robotics field is that robots need to represent and reason about information at different levels of abstraction at the same time.

If the environment is proposed to be represented by local metric maps connected into a topological network. This technique allows the use of maps that are not metrically consistent on the global scale, although they are metrically consistent locally [13].

\section{INSTRUCTED NAVIGATION FOR HUMANOID ROBOTS (INHR)}

In the present section we exemplify some features of the INHR system (Instructed Navigation for Humanoid Robots), which is designed with the purpose of efficient and effective navigation of Fujitsu HOAP-2 Humanoid robots (Humanoid for Open Architecture Platform), with navigation tasks in a miniature city. The miniature city is built on a $5 \mathrm{~m} \times 3.2 \mathrm{~m}$ area which is suitable to the HOAP-2 Humanoid dimensions (50 cm x $25 \mathrm{~cm} \times 16 \mathrm{~cm})$ [15]. Figure 1 shows the layout and the physical realization of our miniature city. Currently, route instructions are communicated to the humanoid by using formalized expressions, called Formal Route Instructions (FRIs) via a Graphical User Interface (GUI).

The system is composed of three main modules as shown in Figure 2. The first module is the route processing module which receives route descriptions from the user and transfers them into topological maps (TM). TMs are connected in the spatial planning stage (module 3) with the output from the vision stage to calculate the Humanoid actions and generate the motion commands. The second module is the vision processing module. It starts with capturing video streams from the Humanoid's cameras, and processes these streams to detect and recognize the landmarks in the miniature city. It is also involved in calculating the distance between robot and the recognized landmark. The final module is the action processing. This module constructs a structured representation of the Humanoid motion commands, to be realized by the Humanoid's actuators.

This paper focuses on the route processing module stages and how to process the route given by the user to generate a topological representation of the spatial environment. The route processing module consists of three main stages. The first is the stage of processing FRIs (Formal Route Instructions), which are expressions in a formalized style used by the human instructor to build route descriptions preventing misunderstanding and ambiguity. In this stage, the route's reference is an intrinsic reference which describes all objects and directions with respect to the Humanoid's body. The intrinsic reference is used to avoid the conversion process between different reference systems (relative, intrinsic, and absolute). The second stage is the CRIL (Conceptual Route Instruction Language) [9] representation which is used to analyze the route description to motion actions, spatial relations, and landmarks. This stage interacts with the landmark's database to retrieve the main features of each landmark described in the route. The final stage is the topological map presentation which is used to translate the CRIL actions to a spatial graph. This graph is used to connect each landmark in the route description with its neighbor landmarks in a network by using route spatial relationships. This graph is submitted to the action processing stage to process the route and generate the Humanoid actions. In the next subsequent sections, the three stages of the route processing module will be elucidated in details. 


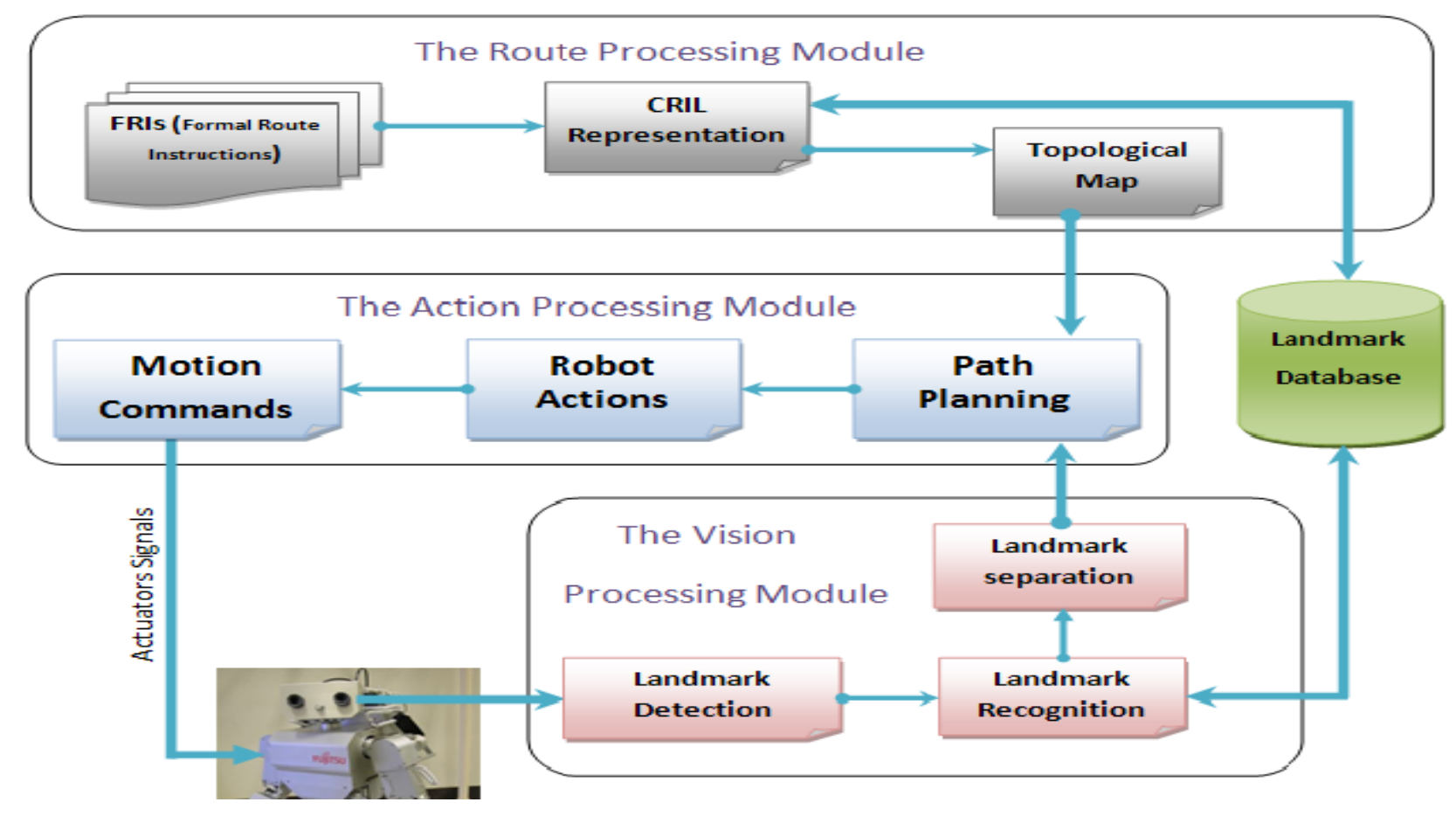

Figure 2. Architecture of the Humanoid Navigation System.

\subsection{The Level of Formal Route Instructions}

FRIs provide elementary instruction statements in a formalized language, which eventually leads to a sequence of INHR-internal commands to be executed by the Action Processing Module. FRI is intended as a semi-formal language to be used by non-expert users via a structured GUI; currently we prepare an empirical test of the usability of the FRI-interface. Each FRI represents an instruction, which relates motion actions with some landmark(s) by use of suitable spatial relationships. The inventory of FRIs containscurrently - three classes of commands; this inventory reflects the inventory of CRIL, which will be described in section 3.2 (see [9], [16]). The first class includes position commands that refer to the current position of the robot. These commands are primarily used to identify the start and end position of the robot by using a spatial preposition and a landmark. They can also be used during the robot route description to describe relevant confirmations of the robot's current position with respect to a landmark. These commands are represented in FRI by using three different commands: \$START(), \$STOP(), and \$BE() as shown in Table 1. The prominent role of landmarks for route instructions is reflected by the syntactical condition that position FRIs have landmarks as obligatory arguments (see column 'syntax' of Table 1; optional arguments are coded via square brackets). The second class is that of locomotion commands. These commands give the robot the order to move to a particular region or to go in a particular direction with respect to certain landmarks. In FRI, this type of commands can be presented by different operators depending on the situation such as: $\$ G O()$, \$CROSS(), \$PASS(), and \$FOLLOW(). The last category is that of change of orientation commands. These commands are used to change the direction of the robot by turning or rotating to a specific direction [16]. \$TURN(), and \$ROTATE() commands are used in FRIs to represent the orientation changes of the robot during the execution of its navigation task.
Additionally, FRIs can be used to build complex instructions that are structured as 'blocks'. Each block begins with a $\$ \mathrm{GO}()$ statement and ends before the next $\$ G O()$ statement-except for the starting and ending statement. All statements in the block are processed in serial and executed as a single sub-route in the Humanoid navigation task.

Table 1 gives an overview of the commands mentioned above exemplified with corresponding verbs, prepositions, and adverbs, which are told to naïve users for introducing them in FRI-usage. For example, $\$ \mathrm{GO}()$ command can be represented by the following syntax:

\section{$\$ G O([$ Count], [Direction] $\mid$ [Pre1], Landmark1, [Pre2],} [Landmark2])

The first parameter in the syntax (Count) presents the number of turns whereas Direction specifies the direction of the turn. Pre represents the formal counterpart to a preposition or an adverb which will be used in the spatial statement. Finally, Landmark specifies the landmark name. The FRI sequence presented in Table 2 is constructed to lead the robot from the railway station to the town hall in the miniature city (cf. the depiction of this route in Figure 1.

In this route, the robot is instructed to begin at a starting point with the railway station to its left. First, the robot has to move to the crossroads, then it is instructed to cross the street, to pass a building on its left, and to move to the next crossroads. Afterwards the robot should turn left, walk down the street until the next crossroads, where it has to turn right. Then he shall walk straight on, pass the Burger King Restaurant to the right, and the C\&A department store to the left, and has to go on until reaching the next crossroads. The next instructions include the robot's crossing of the street, going straight on, passing a church to the left, passing Karstadt department store to the right, and then turning right at the next crossroads. Finally, the robot has to keep walking down the street until it is standing to the right of the town hall, which is determined as its destination. 
Table 1. The FRIs Commands.

\begin{tabular}{|c|c|c|}
\hline $\begin{array}{c}\text { Command } \\
\text { Type }\end{array}$ & $\begin{array}{c}\text { Command } \\
\text { Name }\end{array}$ & Syntax \\
\hline \multirow{3}{*}{ 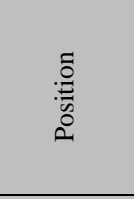 } & \$START() & $\begin{array}{ll}\text { \$START } & \text { ([Pre1|Direction], Landmark1, } \\
& \text { [Pre2], [Landmark2]) }\end{array}$ \\
\hline & \$STOP() & $\begin{array}{ll}\text { \$STOP } & \begin{array}{l}\text { (Pre1|Direction, Landmark1, } \\
\text { [Pre2], [Landmark2]) }\end{array}\end{array}$ \\
\hline & $\$ B E()$ & $\begin{array}{l}\text { \$BE (Pre1|Direction, Landmark1, [Pre2], } \\
\text { [Landmark2]) }\end{array}$ \\
\hline \multirow{4}{*}{ 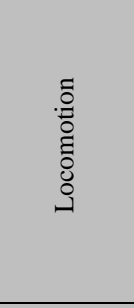 } & $\$ G O()$ & $\begin{array}{ccc}\text { \$GO([Count], [Direction] } & \text { [Pre1], } \\
{[\text { Landmark1], }} & \text { [Pre2], } \\
\text { [Landmark2]) } & \\
\end{array}$ \\
\hline & \$CROSS() & $\begin{array}{ll}\text { \$CROSS } & \text { ([Pre1], Landmark1, [Pre2], } \\
& \text { [Landmark2]) }\end{array}$ \\
\hline & \$PASS() & $\begin{array}{cl}\text { \$PASS } & \text { ([Pre1], Landmark, direction, } \\
& \text { [Pre2], [Landmarket2]) }\end{array}$ \\
\hline & \$FOLLOW() & $\begin{array}{c}\text { \$FOLLOW } \begin{array}{c}\text { ([Landmark1], } \\
\text { Landmark2) }\end{array} \\
\end{array}$ \\
\hline \multirow{2}{*}{ 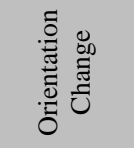 } & \$ROTATE() & \$ROTATE (Direction, Pre, Landmark) \\
\hline & \$TURN() & $\begin{array}{ll}\text { \$TURN } & ([\text { Count }], \quad[\text { Pre1], } \\
& {[\text { Pre2], [Landmark]) }}\end{array}$ \\
\hline
\end{tabular}

Table 2. A FRI route description from railway station to town hall.

\begin{tabular}{|c|l|}
\hline $\begin{array}{c}\text { Block } \\
\text { Number }\end{array}$ & \multicolumn{1}{c|}{ FRI Statement } \\
\hline $\mathbf{1}$ & \$START(RailwayStation, left) \\
\hline $\mathbf{2}$ & \$GO ( to, CrossRoads) \\
\hline $\mathbf{3}$ & $\begin{array}{l}\text { \$GO(forward, into, Street) } \\
\text { \$PASS(Building, left) } \\
\text { \$BE(at, CrossRoads) }\end{array}$ \\
\hline $\mathbf{4}$ & $\begin{array}{l}\text { \$GO ( left, into, Street, to, CrossRoads) } \\
\text { \$TURN (right) }\end{array}$ \\
\hline 5 & $\begin{array}{l}\text { \$GO (forward, into, Street) } \\
\text { \$PASS (BurgerKing, right) } \\
\text { \$PASS (C-and-A, left) } \\
\text { \$BE(at, CrossRoads) }\end{array}$ \\
& $\begin{array}{l}\text { \$GO(forward, into, Street) } \\
\text { \$PASS (Church, left) } \\
\text { \$PASS (KarStadt, right) } \\
\text { \$BE(at, CrossRoads) } \\
\text { \$TURN(right) }\end{array}$ \\
\hline $\mathbf{6}$ & $\begin{array}{l}\text { \$GO (forward, into, Street) } \\
\text { \$STOP(left, TownHall) }\end{array}$ \\
\hline
\end{tabular}

\subsection{CRIL Representation}

Tschander, Schmidtke, Habel, Eschenbach and Kulik [9] proposed the idea of a Geometric Agent that simulates instructed navigation in a virtual planar environment. In their approach Conceptual Route Instruction Language (CRIL), that is kindred to Jackendoff's conception of semantics (see [17], [18]) are used to represent the meaning of natural language route instructions. CRIL-expressions are constructed from a basic inventory of descriptive operators. On the one hand, CRIL-expressions specify the semantics of natural language expressions using methods of formal semantics; in particular CRIL functions as the output of an experimental interface from written instructions in German. On the other hand, CRIL is an internal language, that (1) is the representational medium of spatial reasoning [9], (2) it relates internal models to perceptual objects, and thus (3) specifies actions the Geometric Agent can carry out.

In the INHR system, we use CRIL to transfer FRI instructions to actions and spatial relationships. In CRIL, there exist three types of conceptual entities extracted from route descriptions that are currently in the foreground of the INHR approach. The first type concerns motion actions, which can be considered as CRIL counterparts of verbs of motion. The second type includes spatial concepts, which provide the specification of relations between spatial entities and regions, e.g. those that are used for relating paths and landmarks with each other. The last type concerns landmarks and paths and there distinguished features. These components are considered to be derived from the CRIL implementation. In the following subsections, they will be considered briefly.

\subsubsection{The Inventory of Actions}

Natural language descriptions of motion frequently involve two kinds of expressions that connect to spatial structure [9]: a verb of motion (such as 'go', 'turn', 'enter', ...) and a directional adverb or a directional prepositional phrase (such as 'into the zoo', 'through the park', 'back', 'straight on') [19].

Verbs of "wayfinding relevant actions" are used to indicate the robot behavior during its navigation task. As seen in Table 3, they can be classified into four classes. The first is that of verbs of position, which we use in INHR to represent the current position of the robot. These verbs can be used to identify the start and end position of the robot by using a spatial preposition and a landmark. They can be used also during the robot route description to make confirmation to its orientation. These verbs are represented in CRIL by using the basic concept !BE_AT(x, p), where $\mathrm{x}$ is the navigating agent - in our case the robot-and $p$ is the position. The second group is that of verbs of locomotion. These verbs instruct the navigating agent to move in a particular direction or to a particular region often specified with respect to a certain landmark. In CRIL, verbs of this type are based on the concept !GO(x, w), where $\mathrm{w}$ is the path to be move on. The third group concerns notifying, this means instructing the navigating agent that it has to perceive an object to insure that it is on the correct way. For this type of instructions in CRIL the basic concept !VIEW(x,r) is used. Whereas notifying is common in natural language instructions, explicitly as well as implicitly, the current inventory of FRI does not include this class; since kindred ways of description can be realized by the block structure (see Table 2). The last group concerns verbs of change of orientation. These verbs are used to instruct navigating agents to turn (move on a curved path) or to rotate, i.e. to change the orientation without locomotion. Both subtypes are based on the basic concept !CH_ORIENT(x, d), where $\mathrm{d}$ is a direction of the turn.

Table 3. CRIL Presentations for natural language verbs particularly relevant in route instructions.

\begin{tabular}{|c|c|c|}
\hline \multirow{2}{*}{\begin{tabular}{|} 
Verb Type \\
Position
\end{tabular}} & FRI Commands & CRIL Command \\
\hline & $\begin{array}{c}\text { \$START() } \\
\text { \$BE() } \\
\text { \$STOP() }\end{array}$ & !BE_AT(x, p) \\
\hline Locomotion & $\begin{array}{c}\text { \$GO() } \\
\text { \$CROSS() } \\
\text { \$FOLLOW() } \\
\text { \$PASS() }\end{array}$ & !GO(x, w) \\
\hline Notifying & & !VIEW(x, r) \\
\hline $\begin{array}{l}\text { Change of } \\
\text { orientation }\end{array}$ & $\begin{array}{c}\text { \$ROTATE() } \\
\text { \$TURN() }\end{array}$ & !CH_ORIENT(x, d) \\
\hline
\end{tabular}


Table 4. CRIL implementation of FRIs route

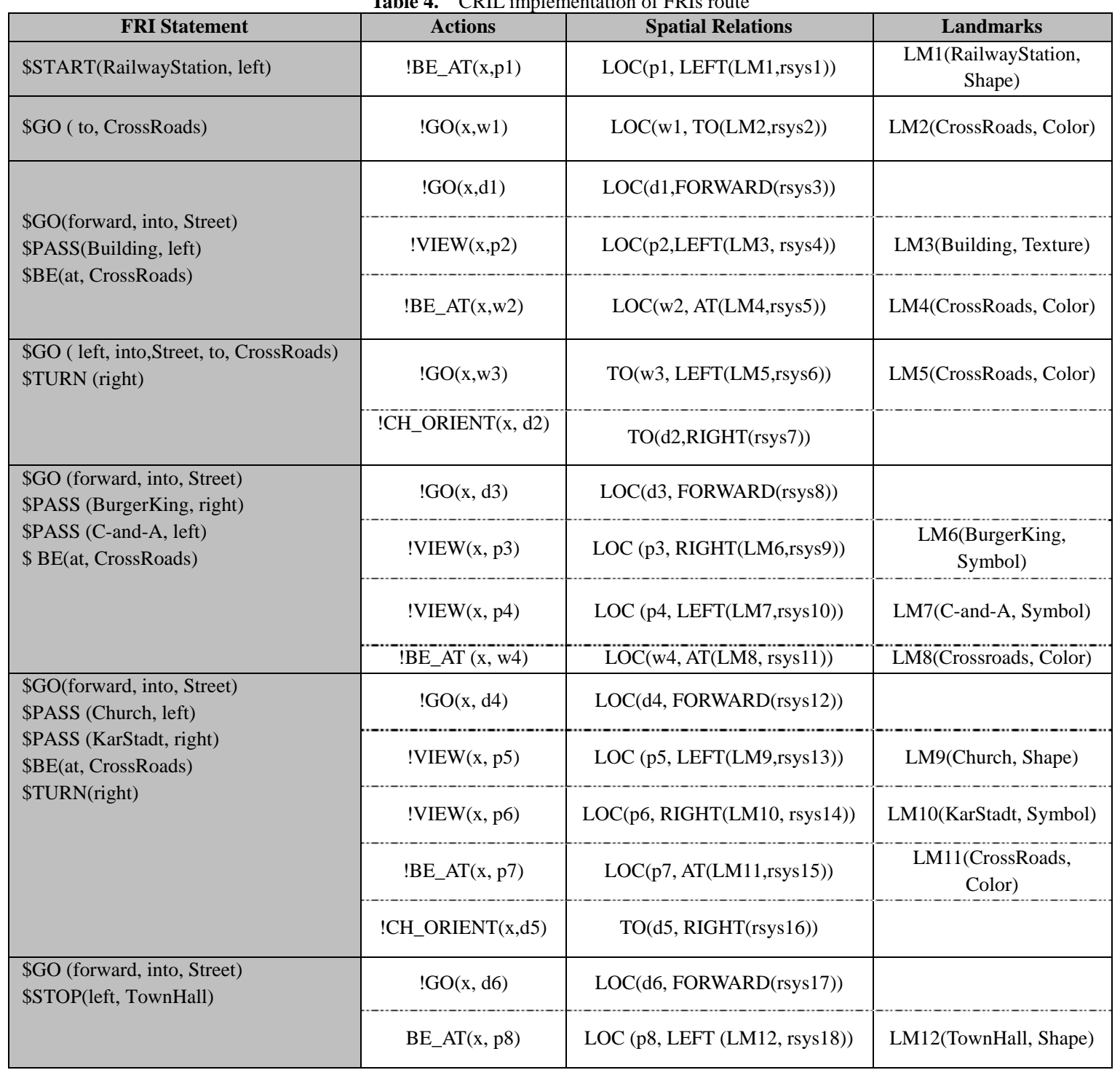

\subsubsection{Spatial Relations}

Spatial prepositions and adverbs are considered as the second kind of expressions of the spatial structure which identifies the spatial relationships. They can be expressed in CRIL by using LOC(w|p, Pre(LM)) syntax, where rsys is a spatial reference system (cf. [9], [16]) or by using the PRE(w ,Pre(LM)) syntax.

On the other hand, the spatial or directional prepositions can be divided into four classes [15]. The first class is the goal prepositions group which specifies the end of the path. The second one is the source prepositions group which gives the start of the path, the third class is the course prepositions group which characterizes the intermediate course of the path, and the final class is the shape prepositions group which identifies the shape of the path.

\subsubsection{Landmarks features}

The third information item that can be extracted from the route description is the landmarks. Landmarks are chosen to indicate the necessity of changing direction or simply confirm correctness of former decisions about the route. The landmarks in our miniature city are identified by using the following four types:
- Shape: The landmark can be recognized by the shape, if its shape has different features and characteristics which can be easily noticed from the other surroundings. These features like the style of the building, contrast, and landmark figure. The railway stations, Town Hall, and churches can be considered as examples of this type.

- Symbol: It is used if the building has a unique symbol or trademark. These symbols are used to recognize the landmarks in the city during the navigation process. Supermarkets, restaurants, and shops can be recognized by using their symbols.

- Texture: Other landmarks can be recognized by their visual properties such as its texture. These landmarks can be recognized if their textures are symmetric and different from the surrounding objects. Houses and buildings can be identified by their textures.

- Color: The landmark color is one of the valuable features which can be used to identify the landmark. We recognize the crossroads in our miniature city by using doted white lines. The street's boundaries are indentified by using black lines. 


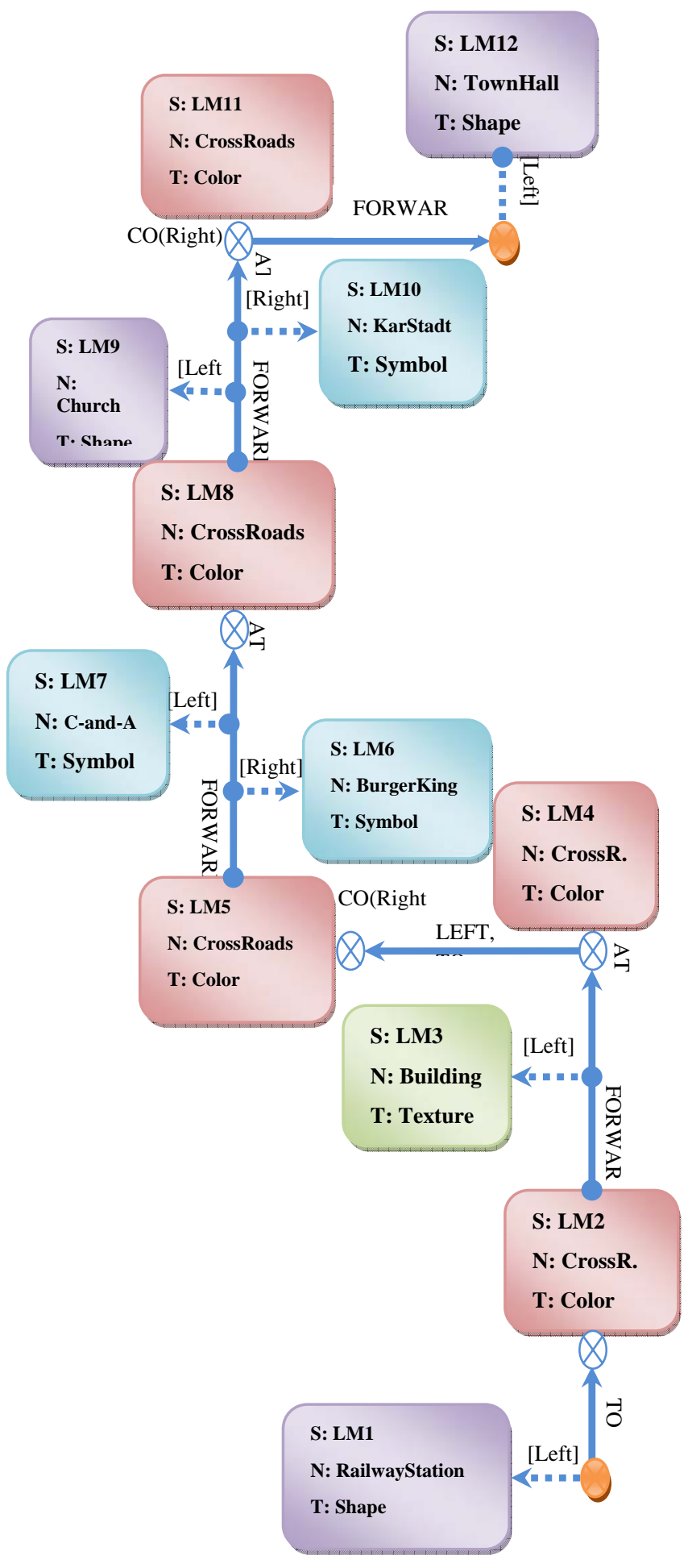

Figure 3. Topological Map presentation of the route (Railway Station to Town Hall).

The landmark is represented in the INHR-adaption of CRIL by using the following syntax:

$$
L M_{i} \text { (Name, type) }
$$

where i presents the landmark number in the route. The Name represents the landmark name,. The type presents the way that used to recognize the landmark. The landmark's features are store in the database and they are retrieved and processed by the CRIL stage.
The CRIL representation of route description from the railway station to the town hall is shown in Table 4. Each FRI is converted to its equivalent CRIL action, spatial relationship, and landmark.

\subsection{Topological Map}

As discussed previously, the topological map is a graph representation of the environment, where nodes correspond to distinct places that can be recognized by robot. It is an abstract representation of the spatial knowledge that describes relationships among features of the environment, without using any absolute reference system. We use the actions, spatial relations, and the landmark features which are extracted from the CRIL representation to build the topological map. Figure 3 shows the topological map of the route from the railway station to the town hall in our miniature city.

This map is consists of seven nodes. The distance between two nodes presents the execution of CRIL actions which result from one processing unit of the FRI statements. The locomotion actions are introduced as solid arrows, the notation actions are presented as doted arrows pointing to the landmark. The position actions are drawn as node. Finally, the change-orientation actions are represented as a written direction beside the node.

Landmarks are introduced as rounded corner rectangles. These rectangles contain the landmark number, the landmark name, and the recognition type. The spatial relations are written above or below the action arrows.

\section{CONCLUSION}

In this paper, we presented a Formal Route Instruction Language (FRIs), which can be used by naïve users to formulate route instructions for robots. It is intended to guide humanoid robots while it navigates through the miniature city. By using FRI route directions misunderstanding and ambiguities can be avoided. On the other hand, the Conceptual Route Instruction Language (CRIL) was customized to extract the main motion actions of humanoids, the spatial relations, and the landmarks used for instructing humanoids. Finally, we represented the data extracted from the CRIL as a topological map. This map gives a graphical depiction of the proposed humanoid's actions and spatial relations. It is also connects the route's landmarks as a network of landmarks to let the humanoid take an overview of the navigation task.

\section{REFERENCES}

[1] H. Inoue, 'Vision Based Robot Behavior: Tools and Testbeds for Real World AI Research', Proceedings of International Joint Conference on Artificial Intelligence (IJCAI), pp. 767-773, (1993).

[2] J. Jeanne, 'Developing Adjustable Walking Patterns for Natural Walking in Humanoid Robots', Final Paper SURF, (2004).

[3] B. Adams, C. Breazeal, R. Brooks, and R. Scassellati, 'Humanoid Robots: A New Kind of Tool', C. Nehaniv, ed., Springer Lecture Notes inArtificial Intelligence, Vol. 1562, Springer-Verlag, Berlin, (1998).

[4] A. Stoica, 'Humanoids for Lunar and Planetary Surface Operations', Proceedings for 2005 5th IEEE-RAS International Conference on Humanoid Robots, p. 345-350, (2005).

[5] D. R. Montello, 'Navigation'. In P. Shah \& A. Miyake (Eds.), The Cambridge handbook of visuospatial thinking. (pp. 257-294). Cambridge: Cambridge University Press, (2005).

[6] L. Litvintseva , T. Tanaka, K. Yamafuji, and V. Ulyanov, 'Intelligence Computing for Direct Human-Robot Communication Using Natural Language and Cognitive Graphics'. IEEE International Symposium on Computational Intelligence in Robotics and Automation (CIRA '97) p. 332, (1997). 
[7] G. Pradel, and P. Hoppenot, 'Symbolic Trajectory Description in Mobile Robotics', Journal of Intelligent and Robotic Systems, Springer Netherlands , Volume 45, Number 2, February, 2006 , P157-180, (2006).

[8] C. Habel, 'Incremental generation of multimodal route instructions', In Natural language generation in spoken and written dialogue, AAAI Spring Symposium 2003. Palo Alto, CA, (2003).

[9] L. B. Tschander, H. Schmidtke, C. Habel, C. Eschenbach, and L. Kulik , 'A Geometric Agent Following Route Instructions', In C. Freksa, W. Brauer, C. Habel \& K.F. Wender (eds.) Spatial Cognition III (pp. 89-111). Springer: Berlin, (2003).

[10] M. MacMahon, 'Marco: A modular architecture for following route instructions'. In: Proc. of AAAI Workshop on modular construction of human-like intelligence. Pittsburgh, PA, AAAI Press, (2005).

[11] S. Werner, B. Krieg-Bruckner, and T. Herrmann, 'Modelling navigational knowledge by route graphs', In Freksa, C., Habel, C., Wender, K., eds.: Spatial Cognition II. Number 1849 in LNAI. Springer 295-317, (2000).

[12] O. Trullier, S. I. Wiener, A. Bertholz, and J.-A. Meyer, 'Biogically based artificial navigation systems: Review and prospects', Progress in Neurobiology, 51, 483-544, (1997).
[13] P. Zavlangas, and S. Tzafestas, 'Integration of Topological and Metric Maps for Indoor Mobile Robot Path Planning and Navigation', Systems Science Journal, (2002)

[14] B. Kuipers, and T. Levitt, "Navigation and mapping in large scale space’, AI Magazine, 9, 25-43, (1988).

[15] Fujitsu Automation Co., Ltd. 'HOAP-2 Instruction Manual', Third Edition, (2004).

[16] H. R. Schmidtke, L. Tschander, C. Eschenbach, and C. Habel, 'Change of orientation'. In van der Zee, Emile \& Slack, Jon (eds.). Representing direction in language and space. (pp. 166-190). Oxford: Oxford University Press, (2003).

[17] R. Jackendoff, 'Semantic structures', Cambridge, MA: MIT-Press, (1990).

[18] R. Jackendoff, 'A Parallel Architecture perspective on language processing', Brain Research, 1146, 2-22, (2007)

[19] C. Eschenbach, L. Tschander, C. Habel, and L. Kulik, 'Lexical Specifications of Paths', In C. Freksa, W. Brauer, C. Habel, \& K.F. Wender (Eds.), Spatial Cognition II (pp. 127-144). Berlin: Springer, (2000). 